\title{
ESTIMACIÓN DE LOS PARÁMETROS GENÉTICOS PARA EL PESO AL NACER Y AL DESTETE EN GANADO BOVINO DE LA RAZA BRAHMAN
}

\section{ESTI MATI ON OF GENETI C PARAMETERS TO DETERMI NE THE BI RTH AND WEANI NG WEI GHT IN BRAHMAN BOVI NES}

\author{
Donicer Montes V, ${ }^{* 1}$ Esp, Oscar Vergara G, ${ }^{2}$ M.Sc, Esperanza Prieto M, ${ }^{1}$ M.Sc, \\ Andrés Rodríguez $P^{3}{ }^{3}$ Zoot.
}

\begin{abstract}
${ }^{1}$ Universidad de Sucre, Facultad de Ciencias Agropecuarias, Departamento de Zootecnia, Grupo Genética y Reproducción Animal, Sincelejo, Colombia. ${ }^{2}$ Universidad de Córdoba, Facultad de Medicina Veterinaria y Zootecnia. Departamento de Ciencias Pecuarias. Montería, Colombia. ${ }^{3}$ Universidad de la Salle. Bogota, Colombia. *Correspondecia: donicermontes@hotmail.com.
\end{abstract}

Recibido: Agosto 31 de 2007; Aceptado: Enero 10 de 2008

\section{RESUMEN}

Objetivo. Estimar la heredabilidad y las correlaciones genéticas, fenotípicas y ambientales para el peso al nacer y al destete en bovinos brahman. Materiales y métodos. Se analizaron 612 datos de peso al nacimiento (PN) y peso al destete (PD) de terneros de la raza cebú brahman, nacidos entre los años 1996 y 2003, datos pertenecientes a las fincas la Pradera y Mundo Nuevo, en el departamento de Sucre (Colombia). Los datos fueron analizados por el sistema MIXED de SAS. V 8.0. El modelo matemático propuesto incluyó los efectos fijos año, época, sexo, número de partos, finca y sugieren el efecto aleatorio de padre para ambas características. Resultados. Se encontró que los efectos finca y padre fueron estadísticamente significativos para el (PN). En cuanto PD, el año fue estadísticamente significativo $(p \leq 0.05)$, los efectos sexo, número de partos y padre resultaron altamente significativos $(p \leq 0.05)$ y los efectos época y finca no significativos $(p \geq 0.05)$. Los estimados de heredabilidad fueron 0.16 y 0.37 para el PN y PD, respectivamente. Los valores de las correlaciones genéticas, fenotípicas y ambientales fueron respectivamente $-0.2,0.14$ y 0.26 . Conclusión. Los valores hallados para la heredabilidad indican que el fenotipo no es un buen indicador de su genotipo, debiendo recurrir para la selección de estos a la prueba de progenie o pedigrí. El valor de las correlaciones genéticas permite afirmar que las crías con mejores pesos al nacimiento no serán las que alcancen mayores pesos al destete.

Palabras clave: Heredabilidad, destete, mejoramiento genético, brahman. 


\section{ABSTRACT}

Objective. To estimate the heritability and genetic environmental and phenotypic correlations of birth and weaning weight in Brahman bovines. Materials and methods. 612 values for birth weight (BW) and weaning weight (WW) were analyzed for Brahman Bovines born form 1996 to 2003. The data derived from "Pradera" and "Mundo Nuevo" farms in Sucre department (Colombia). Data were analyzed using the mixed SAS V 8.0 system. The proposed mathematical model included the fixed effects: year, time, sex, number of calves, and farm and suggested the random effect of bull for both characteristics. Results. It was found that the effects farm and bull were statistically significant for the (BW). For WW, the year was statistically significant $(p \leq 0.05)$; sex, number of calves and bull effects were highly significant $(p \leq 0.05)$ and time and farm effects were not significant $(p \geq 0.05)$. The estimated heritability was 0.16 and 0.37 for BW and WW, respectively. Genetic, phenotypic and environmental correlation values were $-0.2,0.14$ and 0.26 respectively. Conclusions. Values found for heritability indicate that the phenotype is not a good indicator of genotype, and that it is necessary to use tests of progeny or pedigree for selection. The value of the genetic correlations allows the affirmation that calves with better birth weight will not be those that reach higher weights at weaning.

Key words: Heredity, weaning, genetic improvement, brahman.

\section{NTRODUCCIÓN}

En los sistemas de producción de carne, el peso al destete tiene gran importancia, ya que el influye en la determinación de la eficiencia económica de cualquier sistema de producción de bovinos y puede ser recomendado como criterio de selección (1). Un componente importante a evaluar para estimar la rentabilidad en la producción de carne bovina, es el crecimiento de las crías, lo que implica un óptimo crecimiento pre y posdestete, además de la eficiencia reproductiva de la vaca (2). Por otro lado, las características de crecimiento por lo general presentan índices de herencia $\left(\mathrm{h}^{2}\right)$ de valores medios a altos, lo que sugiere que se pueden establecer programas de mejoramiento genético en poblaciones de bovinos de carne (3).

Varios son los estudios donde se ha estimado la heredabilidad para el PN y PD como los reportados por Martins et al (4), Mello et al (5) y Elzo et al (6), quienes encontraron valores de heredabilidad para $P N$ de $0.59 \pm 0.14,0.39$ y 0.26 en ganado Nelore, Canchim y Sanmartinero, respectivamente, y por Montoni et al (7), Manrique et al (8) en ganado Brahman (0.12 \pm 0.07 y 0.48 , respectivamente). Para el PD se han reportado valores de $0.25,0.14$, $0.10 \pm 0.009$ y 0.33 en ganado Nelore, Guzerat, Brahman y Simmental, respectivamente (9-12). Con relación a las correlaciones genéticas igualmente se han realizado varios estimados como el reportado por Montoni et al (9), Geney y Vergara (13) Montes y Pereira (14) y Manrique (8), en ganado Brahman $(0.17,0.93 \pm 0.03,0.40$ \pm 0.17 y 0.41 , respectivamente).

El objetivo del presente trabajo fue estimar la heredabilidad y las correlaciones genotípicas, fenotípicas y ambientales para el peso al nacer y peso al destete en bovinos de la raza cebú Brahman.

\section{MATERI ALES Y MÉTODOS}

Sitio de Estudio. Los datos utilizados pertenecen a la Hacienda "La Pradera" y "Mundo Nuevo", la primera ubicada en el municipio de Sincé, departamento de Sucre, Vereda "Los Limones". Presenta una altura 
sobre el nivel del mar de $50 \mathrm{~m}$ y una temperatura promedio $28^{\circ} \mathrm{C}$, humedad relativa promedio $80 \%$, precipitación media anual de $1076 \mathrm{~mm}$, correspondiente a un periodo de lluvias entre los meses de abril y octubre y un periodo seco de noviembre a marzo. El área de la hacienda es de 333.06 ha, $70 \%$ del terreno es plano y el $30 \%$ restante es ligeramente ondulado. Los pastos predominantes son: Climacuna (Dichantium anulatum), Colosuana (Bothriocloa pertusa), Angleton (Dichantium aristatumL.También se encuentran árboles y arbustos como Matarraton (Gliricidia sepium), Campano (Samanea samam), Acacia forrajera (Leucaena $s p$ ) Gúacimo (Guasuma ulmifolia), Hobo (Spondia mombin) y Totumo (Crecentia cujete).

La segunda (Mundo Nuevo), se encuentra ubicada en el corregimiento del Pueblito municipio de San Onofre, departamento de Sucre, gran parte del terreno comprendido es plano, el cual en los periodos lluviosos es inundable y una pequeña parte es de topografía semi-ondulada. Presenta una temperatura promedio anual de $27^{\circ} \mathrm{C}$, una pluviosidad de 1400 a $1600 \mathrm{~mm}$ anuales marcadas en un periodo lluvioso en los que caen $85 \%$ de las lluvias distribuidas en los meses de mayo a octubre, un periodo seco desde noviembre hasta Abril. La hacienda posee una extensión de tierra de 241 hectáreas distribuidas en 10 potreros con buena disponibilidad de agua y abundantes pastizales en los que predominan el Angleton (Dichantium aristatum) en un $70 \%$ y un $30 \%$ en Colosuana (Bothriocloa pertusa) y Brachiarias (Brachiarias sp). Los potreros son sometidos a rotación con una carga de 1.5 UGG/ ha. Los animales se suplementan con sal mineralizada al $6 \%$; y en época seca con bloques multinutricionales. Además se encuentran algunos árboles forrajeros como Campanos (Samanea saman) y Orejeros (Enterolobium ciclocarpun).

Recolección de información. A partir de los registros consignados en las tarjetas individuales de las vacas se construyó una base de datos que contenía un total de 1050 observaciones. Se estableció una depuración de datos de los cuales se tomaron solo los padres que tuvieran 5 descendientes en adelante, quedando finalmente una base de 612 datos.

Los datos analizados correspondieron a los pesos de terneros nacidos y destetados entre los años 1992 y 2004, los pesos al destete se ajustaron a 270 días de edad.

Analisís de los resultados. Los datos fueron sometidos a un análisis de varianza basado en el modelo estadístico lineal, teniendo en cuenta el efecto aleatorio del reproductor y los efectos fijos: año, época, sexo del ternero, finca y número de parto de la vacas.

El modelo descrito fue:

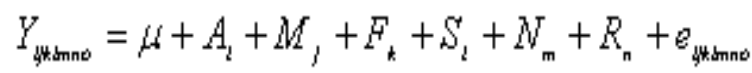

Donde:

$Y_{i j k l m o}=$ Es el peso (al nacer; al destete) del ternero.

$\mu=$ Media general del peso (al nacer o al destete).

$A_{i}=$ Efecto fijo del i-esimo año de nacimiento (destete) del ternero, variando i de 1 a 7.

$M_{j}=$ Efecto fijo de la j-esima época de nacimiento (destete) del ternero, variando $j$ de 1 a 2 siendo: 1 (Seca); 2 (Lluvia).

$\mathrm{F}_{\mathrm{k}}=$ efecto fijo de la k-esima finca donde nació o destetó el ternero, variando $\mathrm{k}$ de 1 a 2

$\mathrm{S}_{\mathrm{I}}=$ efecto fijo del l-esimo sexo del ternero, variando I de 1 a 2 siendo: 1 (macho); 2 (Hembra).

$\mathrm{N}_{\mathrm{m}}=$ efecto fijo del m-esimo parto de la vaca variando $m$ de 1 a 6 siendo: 1 (primero); 2 (segundo); 3 (Tercero); 4 (Cuarto); 5 (Quinto); 6 (Sexto y más).

$\mathrm{R}_{\mathrm{n}}=$ Efecto aleatorio del n-esimo toro

$\mathrm{E}_{\mathrm{ijklmno}}=$ Error experimental

Para la estimación de heredabilidad $\left(\mathrm{h}^{2}\right)$ de las variables dependientes estudiadas (peso al nacimiento y peso al destete) se calcularon los componentes de varianza entre toro y 
dentro de toro y a través de la correlación intraclase entre medios hermanos paternos, utilizando el paquete estadístico Proc Varcomp de SAS V. 8 (15).

$$
h^{2}=\frac{4 \times \sigma^{2} t}{\sigma^{2} t+\sigma^{2} e}
$$

Para el cálculo de las correlaciones, los efectos del reproductor fueron considerados aleatorios para obtener los componentes de varianza de reproductores mediante el programa MIXED DE SAS V 8 (15).

Los análisis de varianza y co-varianza permitieron estimar las correlaciones genéticas ( $r G)$, fenotípica ( $r F$ ) y ambiental (rM), a través la siguiente fórmula:

$$
\begin{aligned}
& r G=\frac{\operatorname{Cov} A(X Y)}{\sqrt{\sigma^{2} A(X) \sigma^{2} A(Y)}} \\
& r F=\frac{\operatorname{Cov} F(X Y)}{\sqrt{\sigma^{2} F(X) \sigma^{2} F(Y)}}
\end{aligned}
$$

$$
r M=\frac{\operatorname{Cov} M(X Y)}{\sqrt{\sigma^{2} M(X) \sigma^{2} M(Y)}}
$$

Donde:

$\operatorname{Cov} A(X Y), \operatorname{Cov} f(X Y)$,

$\operatorname{CovM}(X Y)=$ Componentes de co-varianza genética aditiva, fenotípica y ambiental.

$\mathrm{s}^{2} \mathrm{~A}, \mathrm{~s}^{2} \mathrm{P}, \mathrm{s}^{2} \mathrm{M}=$ Componentes de varianza aditiva, fenotípica y ambiental, respectivamente.

\section{RESULTADOS}

El peso promedio general para el PN fue de $34.88 \pm 3.9 \mathrm{~kg}$, con un coeficiente de variación de $10.83 \%$. En la tabla1 se muestra el análisis de varianza para el PN y PD.

La media hallada para el peso al destete fue

\begin{tabular}{|c|c|c|c|c|c|}
\hline \multirow[b]{2}{*}{$\begin{array}{l}\text { Fuentes de } \\
\text { Variacion }\end{array}$} & \multirow[b]{2}{*}{ G.L } & \multicolumn{2}{|c|}{ Peso al nacer } & \multicolumn{2}{|c|}{ Peso al destete } \\
\hline & & $\begin{array}{l}\text { Cuadrados } \\
\text { medios }\end{array}$ & $p>F$ & Cuadrados medios & $p>F$ \\
\hline Sexo & 1 & 0.0011437 & $0.9929^{\mathrm{NB}}$ & 48492.64 & $<.0001$; \\
\hline Año nacimiento & 7 & 1.118 .427 .988 & $0.3494^{N \Sigma}$ & 12803.85 & $0.096^{*}$ \\
\hline Época nacimiento & 1 & 0.4577796 & $0.8580^{\mathrm{Ns}}$ & 181.25 & $0.677^{\mathrm{Ns}}$ \\
\hline No de partos & 5 & 605.622 .272 & $0.5159^{\mathrm{N}}$ & 21387.17 & $0.001 * * *$ \\
\hline Finca & 1 & 676.313 .866 & $0.0299 *$ & 537.82 & $0.474^{\mathrm{N} \Xi}$ \\
\hline Padre & 34 & 7.827 .225 .644 & $0.0168^{*}$ & 89607.54 & $<.0001$ \% \\
\hline Error & 562 & 8302.13 & & 589671.59 & \\
\hline
\end{tabular}
de $237.08 \pm 35.60 \mathrm{~kg}$, con un coeficiente de variación de $14.42 \%$.

Tabla1. Análisis de varianza para el PN y PD en ganado cebú Brahman.

N.S. no significativo; *significativo $(\mathrm{p} \leq 0.05)$; ** altamente significativo $(\mathrm{p}<0.01)$

Los estimativos de heredabilidad para el PN y PD en ganado cebú Brahman y las correlaciones genéticas, fenotípicas y ambientales, se muestran en las tablas 2 y 3 , respectivamente.
Tabla 2. Estimativos de heredabilidad para el PN y PD en ganado cebú Brahman.

\begin{tabular}{ccc}
\hline Característica & $\mathbf{h}^{2}$ & Error estándar \\
\hline PN & 0.16 & 0.009 \\
PD & 0.37 & 0.009 \\
\hline
\end{tabular}


Tabla 3. Correlaciones genéticas, fenotípicas y ambientales entre el PN PD en ganado cebú Brahman.

\begin{tabular}{cccc}
\hline Correlación & $\begin{array}{c}\text { Genotípica } \\
\text { (rG) }\end{array}$ & $\begin{array}{c}\text { Feno } \\
\text { tipica } \\
\text { (rF) }\end{array}$ & $\begin{array}{c}\text { Ambiental } \\
\text { (rM) }\end{array}$ \\
\hline PN/ PD & $\begin{array}{c}-0.2 \pm \\
0.0317\end{array}$ & 0.14 & 0.26 \\
\hline
\end{tabular}

\section{SCUSI ÓN}

El peso promedio general para el PN fue de $34.88 \pm 3.9 \mathrm{~kg}$. Para las hembras $34.85 \pm 4.1 \mathrm{~kg}$. y para los machos $34.91 \pm 3.7 \mathrm{~kg}$. superando los machos a las hembras en 0.06. El promedio de peso hallado, supera a los encontrados por Ossa et al (16) y Montes y Pereira (14). Resultado superior a los arrojados por este estudio fueron reportados por De Oliveira et al (17) en la misma raza. Aunque el promedio del PN no es bajo, no se podría hacer mucho énfasis para mejorar esta característica, ya que al aumentar el peso al nacimiento probablemente originaría partos distócicos en los dos sistemas de producción.

En la tabla 1 , se puede apreciar que los efectos del año de nacimiento, sexo de la cría, época de nacimiento y numero de partos fueron una fuente de variación no significativa $(p \geq 0.05)$ sobre el PN de los terneros, posiblemente debido a que durante los años estudiados el manejo de las hembras gestantes fue similar, al igual que la rotación de potreros, disponibilidad de forraje, manejo sanitario o posiblemente a la adaptabilidad del ganado Cebú Brahman a las condiciones del trópico bajo colombiano.

Los efectos del reproductor y finca, fueron una fuente de variación significativa sobre la expresión del PN. El padre que produjo crías con mayores PN fue el toro número $130 / 9$ con pesos de $37.85 \mathrm{~kg}$. y el toro con menores pesos en sus crías fue el 0481/9 con pesos de $31.17 \mathrm{~kg}$. superando la progenie del primero a la progenie del segundo toro en $6.68 \mathrm{~kg}$., lo que equivale al $17.64 \%$. Efectos altamente significativos $(p \leq 0.01)$ del reproductor sobre el peso al nacer son corroborados por Arango (18), en la raza Brahman y sus cruces, Montoni et al (7), en la raza Gyr, Ossa et al (16) y Arango (19) en la raza Romosinuano. Geney y Vergara (13), y Montes y Pereira (14) en ganado brahman. El efecto finca, fue una fuente de variación significativa, sobre el PN; siendo el peso para los animales de la finca uno de $32.97 \mathrm{~kg}$, y para la finca dos 35.27 $\mathrm{kg}$, estableciéndose una diferencia de peso entre las dos fincas de $2.3 \mathrm{~kg}$. Esto se debe principalmente a la variación genética que existe entre las fincas y a las diferencias en el manejo alimenticio que tiene cada una de ellas en particular.

La media hallada para el PD fue de $237.08 \pm 35.60 \mathrm{~kg}$. Este se encuentra entre los rangos reportados por Manotti et al (9) el cual esta en 240.19 kg (205 días) en la raza Nelore. A diferencia, otros autores como Manrique et al (8), reporta $210 \mathrm{~kg}$ para la raza Brahman; Montes y Pereira (14), reportan un valor de $228.026 \mathrm{Kg}$; Geney y Vergara (13) reportaron $162.06 \mathrm{Kg}$.

En la tabla 2 se observan las fuentes de variación que influyen sobre el PD, en donde sexo de la cría, número de parto y padre, variables altamente significativas. El año de destete fue una variable significativa $(p \leq 0.05)$, mientras que la época de destete y la finca no fueron variables significativas sobre el PD $(p \geq 0.05)$. Resultados similares con respecto del efecto del sexo sobre el PD de la cría han sido hallados en razas cebuinas por diferentes autores (13, 20-23). Con respecto número de partos de la vaca sobre el peso al destete, en ganado Nelore, se ha encontrado efecto altamente significativo $(p \leq 0.01)(20,24)$.

La diferencia marcada del peso de los terneros al destete con relación al sexo puede ser atribuida al dimorfismo sexual y las diferencias hormonales entre los machos y las hembras. La variación del PD, según el número de partos de la vaca, puede ser atribuida a que el crecimiento predestete de los terneros depende en gran parte de la producción de leche de la madre (habilidad materna), la cual es menor en vacas jóvenes principalmente en primerizas y vacas viejas de noveno y décimo parto (7). 
Los estimativos de heredabilidad para el PN y PD en ganado cebú Brahman se muestran en el tabla 2. El valor de heredabilidad hallado para el PN fue bajo, lo que significa que la variación del peso al nacer en dicha población depende en un $16 \%$ de las variaciones de los genotipos aditivos de los animales y el $84 \%$ al componente genético no aditivo y al ambiente. De acuerdo con el resultado obtenido para la heredabilidad del PN, el método de selección recomendado para mejorar dicha característica sería por pedigrí o progenie. Vergara y Geney (13), y Montes y Pereira (14), encontraron valores de 0.21 y 0.15 respectivamente para el cálculo de heredabilidad del PN, valores que se acercan al reportado en el presente estudio.

Para la heredabilidad del PD se encontró un valor medio, el cual indica que el $37 \%$ de la variación fenotípica es debido a las variaciones genéticas aditivas y el $63 \%$ restante a la genética no aditiva y al ambiente. De acuerdo con el resultado hallado para la heredabilidad del PD, el método de selección recomendado para mejorar dicha característica también sería por progenie o pedigrí. Valores similares a los hallados en el presente trabajo son reportados por Plasse et al (25), Castillo (22) y Montoni et al (7) en las razas Brahman y Nelore quienes hallaron valores de 0.25. Otros autores como Manrique (8), Montes y Pereira (14) y Ossa et al (16) en ganado Brahman reportan valores de 0.45 ; 0.33 y 0.32 respectivamente para la heredabilidad del peso al destete, los cuales se encuentran en el mismo rango hallado en el presente estudio.

Para cualquier programa de mejoramiento genético es necesario evaluar el grado de correlación entre características, ya que estas determinan la relación existente entre ellas, indicando de cierto modo el asocio genético, ambiental y fenotípico en dos o más caracteres. En el tabla 3 se aprecian las correlaciones genotípicas, fenotípicas y ambientales. La correlación genotípica entre el PN y PD es considerada baja y negativa, lo cual demuestra que los genes que influyen o afectan el PN están comprometidos leve y negativamente con el PD, demostrando así que los genes que afectan el PN afectan negativamente el PD: al mejorar el peso al nacer se afecta levemente el peso al destete y viceversa (26). A diferencia de este estudio, De Souza y De Amorin (24) y Martins et al (4) en la raza Nelore encontraron una correlacionan genética entre el PN y PD (0.91 y 0.83, respectivamente). Otros autores como Montes y Pereira (14), Geney y Vergara (13) encontraron valores de 0.40 y 0.93 en la raza cebú Brahman, respectivamente.

La correlación fenotípica obtenida entre PN y PD es considerada baja y positiva, obteniéndose una débil relación entre las dos variables estudiadas. Valores similares al presente estudio son reportados por Montoni et al (7) y Manrique (8) quienes hallaron valores de 0.17 y 0.13 en la raza Brahman respectivamente.

La correlación ambiental entre el PN y PD es media y positiva, sugiriendo que las dos características se encuentran influenciadas por las condiciones ambientales. La correlación fenotípica está principalmente determinada por la correlación ambiental, posiblemente a la heredabilidad baja y media para el PN y PD respectivamente. La correlación ambiental entre el PN y PD hallada en este estudio, concuerda con la reportada por Geney y Vergara (13) quienes hallaron el valor de 0.29 de la correlación ambiental entre el PN y PD en ganado de la raza Cebú Brahman, al igual que Martins et al (4) quienes reportan valor de 0.39 en la raza Nelore.

En conclusión, las grandes discrepancias entre los estimativos obtenidos para estos parámetros genéticos, en Colombia, evidencian la necesidad de estudios más amplios, con poblaciones mayores, y con menos errores en el muestreo. Esto posibilitaría la obtención de estimativos mas exactos. Los resultados obtenidos sirven de base para estimar el valor genético de los animales y con base en éstos elegir a los animales de mayor potencial genético y la eliminación los de menos potencial y de esta manera contribuir a elevar la eficiencia del sistema productivo. 


\section{REFERENCIAS}

1 Ferraz P, De Amorim A, Campos L, De Souza J, Mello M, Mendes C. Tendência Genética dos Efeitos Direto e Materno sobre os Pesos à Desmama e PósDesmama de Bovinos da Raça Tabapuã no Brasil. Rev Bras Zootec 2002; 31(2): 635-640.

2 Martínez G, Bustamante J, Palacios J, Montaño M. Efectos raciales y de heterosis materna Criolla-Guzerat para crecimiento posdestete y características de la canal. Tec Pec Mex 2006; 44(1): 107-118.

3 Cienfuegos Rivas $E$, De Orúe Ríos $M$, Briones Luengo M, Martínez Gonzales J. Estimación del comportamiento productivo y parámetros genéticos de características predestete en bovinos de carne (Bos taurus) y sus cruzas, VIII Región, Chile. Arch Med Vet 2006; 38(1): 69-75.

4 Martins A, Martins F, Lima F, Braga R. Influência de Fatores Genéticos e de Meio sobre o Crescimento de Bovinos da Raça Nelore no Estado do Maranhão. Rev Bras Zootec 2000; 29(1): 103-107.

5 Mello S, Mello M, Da Silva L, Barbosa $T$, Barbosa P. Estimativas de (co)variâncias e tendências genéticas para pesos em um rebanho Canchim. Rev Bras Zootec 2002; 31(3): 17071714.

6 Elzo M, Martínez G, Gonzáles F, Huertas $\mathrm{H}$. Variabilidad y predicciones genéticas aditivas, no aditivas y totales para características de carne en el rebaño multirracial Sanmartinero-Cebú del centro de investigaciones la Libertad. Rev Col Cienc Pec 2003; 16: 49.

7 Montoni D, Rojas G, Verde O, Silva J, Arrionjas M. Producción de un rebaño Brahman bajo condiciones de trópico húmedo .II crecimiento. Rev Fac Agron Maracay 1993; 18: 247-286.
8 Manrique $C$. Parámetros genéticos de la raza Cebú Brahman en Colombia. Resumen. Rev Col Cienc Pec 2003; 16: 48.

9 Mañotti A, Lopez M, Solobodzian A. Parámetros genéticos y ambientales para peso ajustado a 205 días en bovinos de la raza Nelore. Universidad Nacional del Nordeste. Comunicaciones Cientificas y Tecnólogicas. URL Disponible: http:// www.unne.edu.ar/Web/syt/syt/2003/ comunicaciones/04-Veterinarias/V028.pdf; 2003

10 Butarello T, De Oliveira J. Analise genética-cuantitativa do peso aos 8,12 , 18 y 24 meses de idades em um rebanho da raça Guzerat. Rev Bras Zootec 2003; 32(6): 658-665.

11 Castaño J, Mejia A, Quijano J. Heredabilidad y correlaciones entre el peso al destete y a los 18 meses en un hato de ganado de carne. Rev Col Cienc Pec 2003; 16: 48

12 Rosales J, Elzo M, Montaño M, Vega E. Parámetros y tendencias genéticas para características de crecimiento predestete en la población Mexicana de Simmental. Tec Pec Mex 2003; 42(2): 171-180.

13 Geney P, Vergara O. Estimación de los para metros genéticos en bovinos de la raza Cebú. [Tesis Zootecnista]. Sincelejo: Universidad de Sucre. Facultad de Ciencias Agropecuarias; 1999.

14 Montes D, Pereira N. Determinación de parámetros genéticos en algunas características productivas en ganado Cebú Brahman. [Tesis zootecnista]. Sincelejo: Universidad de Sucre. Facultad de Ciencias Agropecuarias; 1999.

15 SAS. SAS/STAT User's Guide (Relase 8.0), Cary, NC, USA: SAS inst Inc; 2001.

16 Ossa G, Manrrique C, Torregroza L. Como utilizar los registros para evaluar animales en la finca. La investigación pecuaria regional Corpoica 1998; 2: 103-108 
17 De Oliveira J, Duarte F, Lobo R. Estudo do desenvolvimento ponderal de bovinos da raça Canchim. Influencia do ambiente. Rev Soc Bras Zootec 1984; 13: 30-39.

18 Arango J. Estúdio genético de características de crecimiento em brahman y sus cruces com Nelore y Guzerat. [Tesis Maestría]. Venezuela: Maestría en producción Animal, Universidad Central de Venezuela, Facultad de Agronomia y Ciencias Veterinarias; 1994.

19 Arango L. Heredabilidad y correlaciones entre el peso al nacimiento, destete y dieciséis meses en la raza Romosinuano. [Trabajo de pregrado]. Colombia: Programa de Zootecnia. Universidad Nacional de Colombia, Facultad de Ciencias Agropecuarias; 1995.

20 Tonhati H, Giannoni M, Duarte A. Avaliacao de parámetros ambientais e genéticos na producao de bovinos da raca Nelore. Fase de aleitamento. Rev Soc Bras Zoot 1986; 15(6): 499-505.

21 Segura J. Comportamiento hasta el destete de un hato Cebú comercial en el sureste de México. Livestock Research for Rural Development 2004; 16: 142159.
22 Castillo S. Factores genéticos y ambientales de características productivas y reproductivas de un hato Brahman bajo condiciones de trópico seco. [Tesis postgrado] Mexico: Universidad Autónoma de Tamaulipas, Faculta de Agronomía, Maestría en ciencias de producción de animales tropicales. URL Disponible en: Uamac$\mathrm{p}$ o s t g r a d o. u a t. m x / alumnos.asp?.pas=MCPAT; 1993.

23 Mascioli A, Paro C, Mello M, Barboza P, De Sena M, Pereira A. Influencia de factores de meio sobre peso de animais da raça Canchim. Rev Soc Bras Zootec 1997; 25(5): 853-865.

24 De Souza J, De Amorin A. Efeitos de factores genéticos e do meio sobre os pesos de bovinos da raça Nelore. Rev Soc Bras Zootec 1995; 24(1): 164-172.

25 Plasse D, Arango J, Fossi H, Camaripano L, Llamozas G, Pierre A, Romero R. Genetic and non-genetic trends for calf weights in a Bos indicus herd upgraded to pedigree Brahman. Livestock Research for Rural Development 2004; 16: 142-159.

26 Pulgaron P, Del Cueto C. Manual de genética y mejoramiento animal. La Habana, Cuba: Editorial Pueblo y Educación; 1995. 